\title{
Développer les compétences syntaxiques et sémantiques des élèves à l'écrit, ou comment enseigner autrement la réécriture à l'école primaire et au collège français ?
}

\author{
Nathalie Rossi-Gensane ${ }^{1 *}$, et Véronique Paolacci $^{2}$ \\ ${ }^{1}$ Université Lyon 2 - ICAR (UMR 5191); ICAR, ENS de Lyon, 15 parvis René Descartes, BP 7000, \\ 69342 Lyon Cedex 07, France \\ ${ }^{2}$ INSPÉ de Toulouse Occitanie-Pyrénées, Université Toulouse 2 Jean Jaurès - CLLE-ERSS (UMR \\ 5260) ; INSPÉ, 181 avenue de Muret, 31076 Toulouse Cedex, France
}

\begin{abstract}
Résumé. Savoir rédiger un texte est l'un des objectifs centraux de l'enseignement du français de l'école primaire et du collège selon les Programmes du Ministère de l'Éducation Nationale (2018). Dans notre article, nous nous sommes attachées à certains aspects syntaxiques et sémantiques de la production écrite chez les élèves français de fin d'école primaire et ceux de collège. En effet, les enseignants se heurtent à la difficulté des élèves à mettre en œuvre une segmentation en phrases en ayant pris conscience de la nécessité de la ponctuation (en réception et en production), d'une part, et à appréhender la cohérence et la cohésion textuelles, d'autre part, non seulement dans le premier état de leur écrit, mais aussi dans la phase de réécriture. Selon notre hypothèse de travail, amener les élèves à penser la posture de lecteur leur permet de penser autrement la posture de scripteur. Cette posture de lecteur peut en outre être renforcée par une posture d'évaluateur-prescripteur, qui incite les élèves à exprimer le ressenti du lecteur par des commentaires métalinguistiques. Nous rendons compte dans notre article des premiers résultats d'une recherche collaborative qui, à partir d'une ingénierie didactique mise en œuvre dans huit classes d'école primaire et de collège, a souligné l'intérêt d'enseigner explicitement les phénomènes de cohésion textuelle (en particulier la ponctuation) dans une séquence d'écriture/réécriture. Les réécritures d'un même texte accompagnées par des conseils d'élèves seront comparées avec un corpus d'écrits émettant les mêmes consignes en provenance d'étudiants de Master 1 se destinant à devenir professeurs des écoles.
\end{abstract}

\begin{abstract}
Improving pupils' syntactic and semantic writing skills, or how to teach rewriting differently in French primary and secondary school? According to the curricula of the French Ministry of National Education (2018), French-language teaching in French primary and secondary school assigns a central place to text writing. This article is focused on certain syntactic and semantic aspects of the production of texts
\end{abstract}

\footnotetext{
* Corresponding author : nathalie.rossi-gensane@univ-lyon2.fr
} 
by French pupils at the end of primary school and in the first four grades of secondary school. As is observed by teachers, pupils encounter difficulties in implementing sentence segmentation after becoming aware of the central part played by punctuation (both in reception and in production), on the one hand, and in handling text coherence and cohesion, on the other hand, not only in the first draft, but also while rewriting. According to our working hypothesis, leading pupils to think about the part of the reader enables them to think differently about the part of the writer. In addition, the part of the reader can be reinforced by making pupils play the part of an examiner, which induces them to express by metalinguistic comments what the reader feels. This article presents the first results of a research taking into account eight primary and secondary school classes, which has pinpointed the relevance of teaching text cohesion (in particular, punctuation) explicitly in a pedagogical sequence of text writing/rewriting. The rewriting of the same text accompanied by the advice of pupils will be compared with a corpus of similar texts produced by students in the first year of a Master programme in order to be primary school teachers.

\section{Introduction ${ }^{1}$}

Savoir rédiger un texte est l'un des objectifs centraux de l'enseignement du français de l'école primaire et du collège français. Selon les Programmes du Ministère de l'Éducation Nationale (2018), les connaissances et compétences visées sont les suivantes en cycle 3 , qui comprend les deux dernières années d'école primaire et la première année de collège (élèves de 9 à 12 ans) :

[Pour] rédiger des écrits variés, [il faut :]

- mettre en ouvre (de manière guidée, puis autonome) une démarche de rédaction de textes : convoquer un univers de référence, un matériau linguistique (lexique et syntaxe déjà connus ou préparés pour l'écrit demandé), trouver et organiser des idées, élaborer des phrases, les enchaîner avec cohérence, élaborer des paragraphes ou d'autres formes d'organisation textuelles,

- mobiliser des outils liés à l'étude de la langue à disposition dans la classe (matériau linguistique, outils orthographiques, guides de relecture, dictionnaires en ligne, traitements de texte, correcteurs orthographiques) ;

- mobiliser ses connaissances sur la langue (mémoire orthographique des mots, règles d'accord, ponctuation, organisateurs du discours, etc.) [...]. (MEN, Cycle 3, 2018 : 17)

Ce sont des objectifs ambitieux qui recouvrent les nombreuses compétences rédactionnelles attendues pour écrire un texte cohérent et cohésif. Dans la recherche présentée dans cet article, nous nous sommes attachées à certains aspects syntaxiques et sémantiques de la production écrite chez les élèves français de fin d'école primaire et ceux de collège : nous nous penchons sur le troisième point exposé ci-dessus concernant les « connaissances sur la langue ». En effet, les enseignants que nous croisons au cours de nos formations aboutissent au même consensus : pour les élèves, il est difficile d'appréhender la cohérence et la cohésion textuelles ${ }^{2}$ non seulement dans le premier état de leur écrit, mais aussi dans la phase de réécriture, cette dernière phase étant liée à l'évaluation de l'enseignant.

À partir de ces constats, plusieurs questions peuvent être posées : comment inciter les élèves à porter leur attention sur la syntaxe des phrases ou, plus précisément, la segmentation des textes en phrases ? Comment développer leur sensibilité syntaxique en insistant sur l'importance de la ponctuation (en réception et en production) ? Comment développer leur sensibilité sémantique en soulignant la nécessité de la mention préalable de référents pour les expressions anaphoriques ? Plus généralement, comment accroître chez 
les élèves la prise en compte du lecteur potentiel quand ils écrivent ? Comment les conduire à réécrire en considérant tous ces points?

Selon notre hypothèse de travail, amener les élèves à penser la posture de lecteur leur permet de penser autrement la posture de scripteur. Cette posture de lecteur peut en outre être renforcée par une posture d'évaluateur-prescripteur, qui incite les élèves à expliciter le ressenti du lecteur par des commentaires métalinguistiques. Présente dans les compléments aux programmes scolaires du Ministère français de l'Éducation Nationale, la fiche d'Éduscol $^{3}$ (2015:2) Réécrire : principes et tactiques développe ces aspects qui nous ont inspirées. Il s'agit de privilégier la lecture et l'analyse des textes entre pairs :

Ces moments permettent aux élèves de mesurer leurs réussites et leurs faiblesses - de prendre conscience d'écarts et d'adopter une attitude réflexive à l'égard de l'écriture. Ils marquent également une véritable entrée en littérature en ce qu'ils mettent l'accent sur l'acte de réception et donc, sur les effets du texte.

Nous détaillons dans cet article les premiers résultats d'une recherche collaborative menée à l'Institut National Supérieur du Professorat et de l'Éducation (désormais INSPÉ) de Toulouse Occitanie-Pyrénées. Dans un premier temps, nous brosserons quelques propos généraux concernant la didactique de l'écriture. Puis nous présenterons la recherche dont sont issues nos analyses, de même que les principes méthodologiques retenus. Nous exposerons enfin quelques résultats saillants pour conclure sur les intérêts didactiques de ce type de travaux.

\section{1 Écrire et réécrire à l'école et au collège}

L'apprentissage de l'écriture est complexe comme le rappelle le Cnesco (Conseil national d'évaluation du système scolaire) dans son rapport de 2018. Pour les jeunes apprenants, le geste graphique peut être un premier obstacle à franchir. Il existe également la difficulté de la mise en mots aux niveaux orthographique, lexical et grammatical. Ces étapes sont combinées avec, du point de vue de l'enseignant, la nécessité d'accompagner les élèves dans la planification, la production et la révision des textes afin d'encourager une réécriture efficiente. Il s'agit de travailler, mais aussi de retravailler, les écrits, ce qui questionne l'enseignement de la réécriture, selon le rapport du Cnesco (2018), et, en particulier, l'enseignement des opérations, mises au jour par les travaux en génétique de l'écriture, d'ajout, de suppression, de remplacement et de déplacement (Fabre-Cols, 2002).

Crinon et Marin (2014: 24) revendiquent un enseignement raisonné de la production écrite en soulignant la prise en compte du lecteur potentiel par le jeune scripteur :

La construction d'une représentation mentale de son projet d'auteur et de la manière dont le texte sera lu est un apprentissage essentiel dans le long trajet de l'apprenti scripteur.

Nous insistons sur l'expression "la manière dont le texte sera lu », car ce type de remarque est fondateur de nos objectifs didactiques. Les compétences syntaxiques doivent notamment se développer; il est néanmoins stérile d'en rester à la phrase de base (Crinon \& Marin, 2014 : 18). Il est plus efficace de partir d'emprunts à la fois de textes d'auteurs et de textes de pairs (Cautela \& Marin, 2013), les didacticiens promouvant la lecture entre élèves.

\subsection{La segmentation en phrases}

La segmentation en phrases est une compétence longue à acquérir. Nous avons montré dans nos précédents travaux (Paolacci \& Rossi-Gensane, 2012; Paolacci \& Rossi-Gensane, 2014 ; Paolacci \& Rossi-Gensane, 2016; Rossi-Gensane \& Paolacci, 2016; RossiGensane, Roubaud \& Paolacci, 2019) que les images de la phrase dans les textes d'élèves pouvaient être multiples, dans la mesure où la phrase ne se réduit pas à la démarcation traditionnelle «majuscule-point». En effet, la phrase ainsi définie, qui peut être appelée 
graphique, gagne à être mise en regard avec une autre phrase, fondée sur un critère syntaxique, qui correspond à « une structure constituée d'un [pivot] ${ }^{4}$ et de l'ensemble des rapports de dépendance contractés autour de ce [pivot], directement [...] ou indirectement » (Feuillard, 1989: 214). Le pivot est « un noyau relationnel et non sémantique, ce qui signifie [qu'il] n'est pas forcément l'élément le plus important du point de vue du sens, mais celui auquel se rattachent les divers constituants d'une structure [...]» (Feuillard, 1989 : 65). En outre, le pivot n'est pas obligatoirement verbal, comme, par exemple, dans Délicieux, ce gâteau!, où il est adjectival. Le recours à la phrase syntaxique met en évidence l'emplacement de bornes de natures diverses à nécessairement matérialiser : signes de ponctuation forte, si la fin de la phrase syntaxique se confond avec la fin d'une phrase graphique; signes de ponctuation faible ou moyenne, connecteurs, si la fin de la phrase syntaxique et la fin de la phrase graphique ne coïncident pas. Dans le dernier cas, deux phrases syntaxiques peuvent par exemple être séparées par une virgule, qui sera dite interphrastique (à la différence des virgules opérant une démarcation à l'intérieur d'une phrase syntaxique, qui seront dites intraphrastiques) ${ }^{5}$.

\subsection{Un enseignement de la ponctuation au service de l'écriture/lecture des textes}

À ce jour, la didactique de la ponctuation a été l'objet de nombreux travaux et études (par exemple, David \& Vaudrey-Luigi, 2014 ; Paolacci \& Rossi-Gensane, 2014; Paolacci, Dufour \& Bain, 2016 ${ }^{6}$ ). Les linguistes et les didacticiens soulignent que les signes de ponctuation font système et que ce système est plurifonctionnel. On peut en effet considérer que ces signes ont des fonctions syntaxique et sémantique liées à une fonction énonciative. Catach (1994) mentionne également la fonction intonative, très présente dans les discours scolaires, comme nous le verrons infra en 3.1 et en 4.1. Cependant, il n'est pas rare d'observer dans les classes (comme nous en sommes témoins dans les écoles que nous visitons) des séances consacrées de manière isolée à la ponctuation, où cette dernière se réduit parfois à un catalogue de signes, conformément à l'approche de certains manuels de grammaire scolaires. Or, les savoirs déclaratifs que les élèves peuvent acquérir dans leur cursus ne sont d'aucune aide quand il faut les réinvestir en savoirs procéduraux, en particulier dans l'acte d'écrire. Ces problématiques se retrouvent pour les écrits d'étudiants (Niwese, Lafont-Terranova \& Jaubert, 2019), notamment pour les emplois de la virgule que nous avions analysés dans une étude antérieure (Paolacci \& Rossi-Gensane, 2019). Au regard de ces différents aspects, nous allons présenter la méthodologie de la recherche qui est au cœur de notre travail.

\section{Choix méthodologiques dans le cadre d'une recherche collaborative}

Notre article trouve sa source dans les premiers résultats d'une recherche collaborative menée sur l'INSPÉ de Toulouse Occitanie-Pyrénées de 2018 à 2020. Ce type de recherche est fondé sur le travail conjoint entre des chercheurs, des formateurs (formateurs des instituts de formation des enseignants (INSPÉ) ou maîtres-formateurs) et des enseignants ayant une classe en responsabilité. La recherche collaborative dont nous rendons compte a été initiée par C. Garcia-Debanc et est animée par l'une de nous; elle interroge les liens entre l'étude de la langue et l'écriture dans la liaison école primaire/collège. L'équipe comporte deux professeurs des écoles (une enseignante ayant une classe rurale à trois niveaux (CE2, CM1, CM2) et un enseignant ayant un CM2), deux professeurs de lettres de collège (ayant en tout quatre classes de $6^{\text {ème }}$, une classe de $4^{\text {ème }}$ et une classe de $3^{\text {ème}}$ ), une conseillère pédagogique et deux formatrices INSPÉ ${ }^{7}$. 
Selon nous, l'enseignement explicite de la ponctuation peut avoir un effet sur la réception de l'écrit et, à plus long terme, sur l'acte d'écriture lui-même. Grâce à cette recherche collaborative, encore en cours au moment où nous écrivons ces lignes, a été mise en place une ingénierie d'enseignement constituée par les étapes que nous présentons dans le tableau ci-dessous :

Tableau 1. Les étapes de la séquence d'écriture-réécriture

\begin{tabular}{|c|c|}
\hline Phase 1 & $\begin{array}{l}\text {-Écriture individuelle de la } \\
\text { consigne des «trois phrases » } \\
\text { (voir ci-dessous) }\end{array}$ \\
\hline Phase 2 & $\begin{array}{c}\text {-Écrit de travail }{ }^{8} \text { sur les signes de } \\
\text { ponctuation et échanges } \\
\text { collectifs : citer tous les signes } \\
\text { connus, les classer et répondre } \\
\text { à la question « À quoi servent } \\
\text { les signes de ponctuation?»» }\end{array}$ \\
\hline Phase 3 & $\begin{array}{l}\text { - Réécriture de l'incipit d'un écrit } \\
\text { d'élève de } 6^{\text {ème }} \\
\text {-Écriture de conseils de réécriture à } \\
\text { cet élève }\end{array}$ \\
\hline Phase 4 & $\begin{array}{c}\text { - Retour sur l'écrit de travail } \\
\text { concernant les signes de } \\
\text { ponctuation et constitution } \\
\text { d'un écrit-bilan collectif }\end{array}$ \\
\hline $\begin{array}{c}\text { Phase } 5 \text { (décalée dans } \\
\text { le temps) }\end{array}$ & $\begin{array}{l}\text {-Lors d'une autre situation } \\
\text { d'écriture, écriture de conseils } \\
\text { à l'un de ses pairs }\end{array}$ \\
\hline
\end{tabular}

Nous nous intéressons dans le cadre imparti à notre article à la phase 3 de la séquence dans une visée comparative. Les élèves devaient réécrire l'incipit du texte d'un élève ${ }^{9}$ de $6^{\text {ème }}$ en ajoutant des conseils de réécriture destinés à cet élève rédacteur.

\subsection{La consigne d'écriture de la copie observée: une tâche d'écriture contrainte}

Nous avons proposé aux élèves de la recherche collaborative ${ }^{10}$ (soit 150 élèves au total) un texte d'un élève de $6^{\text {ème }}$ (élève de 12 ans) qui répondait à une consigne d'écriture contrainte imaginée par C. Garcia-Debanc ${ }^{11}$. Nous avons aussi proposé la copie de $6^{\text {ème }}$ à 55 étudiants de Master 1 MEEF (Master de l'Enseignement, de l'Éducation et de la Formation) de l'INSPÉ de Toulouse se destinant à devenir professeurs des écoles ${ }^{12}$. Notre objectif était de comparer les réécritures d'élèves, âgés de 8 à 13 ans, d'école primaire et de collège en fin de cycle 2, en cycle 3 et en cycle 4 (plus précisément en CE2, CM1 et CM2 à l'école primaire et en $6^{\text {eme }}$ et $4^{\text {ème }}$ au collège). Ces données ont été également comparées aux productions des étudiants se destinant à devenir professeurs des écoles.

La consigne d'écriture était la suivante :

Racontez une histoire dans laquelle vous insérerez, séparément et dans l'ordre donné, les trois phrases suivantes :

Elle habitait dans cette maison depuis longtemps.

Il se retourna en entendant ce grand bruit.

Depuis cette aventure, les enfants ne sortent plus la nuit. ${ }^{13}$

Ces phrases induisent plusieurs problèmes d'écriture qui concernent notamment la cohérence et la cohésion textuelles. Si le syntagme nominal défini les enfants indique avant tout un groupe d'au moins deux membres catégorisés comme "enfants » et «seuls d'un certain type » (Charolles, 2002: 103), les substituts pronominaux elle et il imposent $a$ 
minima un personnage féminin et un personnage masculin (certes sans autre précision). Les syntagmes nominaux démonstratifs cette maison et ce grand bruit supposent que, dans le texte, ils soient précédés de la mention des référents auxquels ils se rapportent. Il convient en outre de signaler que les déterminants démonstratifs ont la spécificité de pouvoir « romp[re] avec le contexte intellectuel dans lequel intervient l'acte de référence, [ce qui] permet de mettre dans le focus d'attention des destinataires un référent qui ne l'était pas jusque-là ou de le présenter sous un jour inédit » (Charolles, 2002: 137). On pourrait également ajouter que la dernière phrase, d'une part avec le syntagme nominal cette aventure formé d'un déterminant démonstratif et d'un nom abstrait, laissant augurer une anaphore conceptuelle ${ }^{14}$, et d'autre part avec le présent à valeur habituelle, s'apparente à une morale de l'histoire et a un statut conclusif. La consigne a été proposée aux élèves des classes des professeurs de la recherche collaborative avant que ne soit travaillée la copie sélectionnée pour la phase 3 de la séquence. C'est cette copie que nous présentons maintenant.

\subsection{La copie de $6^{\text {ème }}$ observée : l'analyse de l'incipit du texte}

La copie de l'élève de $6^{\text {ème }}$ sélectionnée pour la recherche est jointe en annexe dans son intégralité. Les erreurs d'orthographe ont été corrigées afin que les élèves et les étudiants ne se polarisent pas sur ces erreurs dites de surface. Nous nous sommes restreintes à l'incipit du texte, nous arrêtant immédiatement après la deuxième phrase imposée (autrement dit, la troisième phrase imposée n'a pas été prise en compte) :

Il était une fois une jeune fille qui s'appelait Jenny elle habitait dans cette maison depuis longtemps. Jusqu'au jour où elle rencontra un petit garçon prénommé Matthieu ils passaient beaucoup de temps ensemble. Un jour Jenny et Matthieu allaient jouer dehors en pleine nuit, ils allèrent dans un bois soudain il se retourna en entendant ce grand bruit.

Notons que l'élève observé a détourné la consigne en ôtant la majuscule du début des phrases imposées. Le tableau ci-dessous présente des éléments d'analyse syntaxique et sémantique du texte. Les chiffres correspondent au nombre de phrases graphiques désignées par PG1, PG2 et PG3. Les doubles barres délimitent à droite ce que nous avons appelé les phrases syntaxiques.

Tableau 2. Découpage du texte de $6^{\text {ème }}$ en phrases et analyse

\begin{tabular}{|c|c|}
\hline Début du texte & $\begin{array}{c}\text { Éléments d'analyse syntaxique et } \\
\text { sémantique }\end{array}$ \\
\hline $\begin{array}{l}\text { [Il était une fois une jeune } \\
\text { fille qui s'appelait Jenny // } \\
\text { elle habitait dans cette } \\
\text { maison depuis longtemps. //] }\end{array}$ & $\begin{array}{l}\text {-Aucune borne entre les deux phrases } \\
\text { syntaxiques } \\
\text {-Absence de référent pour cette maison }\end{array}$ \\
\hline $\begin{array}{l}\text { [Jusqu'au jour où elle } \\
\text { rencontra un petit garçon } \\
\text { prénommé Matthieu // ils } \\
\text { passaient beaucoup de temps } \\
\text { ensemble. //] PG2 }\end{array}$ & $\begin{array}{l}\text {-Problème de l'apparent complément } \\
\text { circonstanciel de temps débutant par } \\
\text { jusqu'au jour: si complément il y a, à } \\
\text { quoi se rattache-t-il? } \\
\text {-Aucune borne entre les deux phrases } \\
\text { syntaxiques (voir ci-dessous) }\end{array}$ \\
\hline $\begin{array}{l}\text { [Un jour Jenny et Matthieu } \\
\text { allaient jouer dehors en } \\
\text { pleine nuit, // ils allèrent } \\
\text { dans un bois // soudain il se } \\
\text { retourna en entendant ce } \\
\text { grand bruit. //] PG3 }\end{array}$ & $\begin{array}{l}\text { - Deux absences de virgules } \\
\text { intraphrastiques (alors qu'il est } \\
\text { d'usage de délimiter à droite par une } \\
\text { virgule les compléments frontaux) } \\
\text {-Présence d'une virgule interphrastique } \\
\text { - Temps verbal : imparfait de allaient } \\
\text { (jouer) à la place du passé simple }\end{array}$ \\
\hline
\end{tabular}




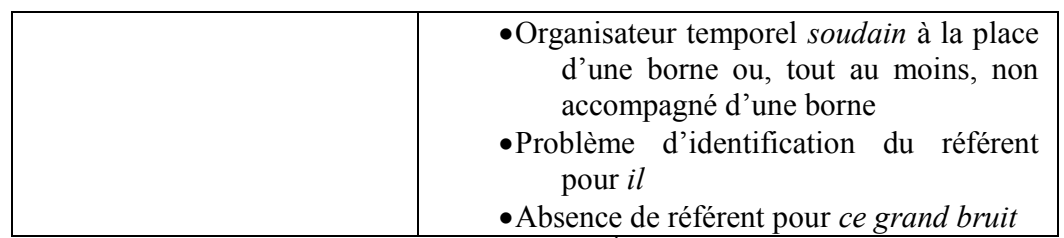

Nous pouvons en conclure que la copie de $6^{\text {eme }}$ met en évidence des problèmes de cohérence et cohésion textuelles, en particulier un phénomène de sous-ponctuation (Béguelin, 2000). Sur un plan sémantique, l'opposition entre imparfait et passé simple paraît mal maîtrisée. Dans la première phrase syntaxique de PG3, l'organisateur temporel Un jour fournit un nouveau point de référence plus volontiers associé à l'emploi du passé simple (allèrent jouer, et non allaient jouer) (voir par exemple Vetters, 1993). La différenciation problématique entre imparfait et passé simple a aussi des répercussions sur la segmentation en début de PG2. Ainsi, alors que, sur un plan syntaxique, le syntagme débutant par Jusqu'au jour serait tout à fait susceptible d'être rattaché au verbe " habiter » en tant que complément circonstanciel de temps, il n'en est pas de même à un niveau sémantique car cet apparent complément, faussement symétrique du complément circonstanciel de temps immédiatement antérieur introduit par depuis, "indique que l'événement en question est saisi dans sa globalité et exclut donc l'imparfait » (Vetters, 1993 : 15) au profit du passé simple, qui exprime un aspect perfectif : ??Elle habitait dans cette maison [...] jusqu'au jour où elle rencontra un petit garçon prénommé Matthieu vs Elle habita dans cette maison [...] jusqu'au jour où elle rencontra un petit garçon prénommé Matthieu. Signalons que le complément circonstanciel de temps depuis longtemps, qui met l'accent sur le déroulement, se comporte à cet égard de manière opposée en imposant une lecture imperfective : Elle habitait dans cette maison depuis longtemps vs *Elle habita dans cette maison depuis longtemps. Au début de PG2, syntaxe et sémantique entrent donc en conflit, ce dernier niveau empêchant de considérer le syntagme débutant par Jusqu'au jour comme un complément différé du pivot verbal habitait, phénomène parfois également dénommé « épexégèse » (Bally, 1944), ou encore « ajout après le point » ou " construction en annexe » (voir par exemple Gautier, 2010). En conséquence, la fin de PG1 coïncide dans ce cas, en l'absence de complément différé, avec une fin de phrase syntaxique (et le syntagme prépositionnel, au début de PG2, correspond à une phrase syntaxique).

\subsection{Réécritures et conseils de réécriture dans le corpus collecté}

Nous avons fait le choix, dans le cadre contraint de notre article, de nous centrer sur la réécriture du début du texte de la copie de $6^{\text {ème }}$ effectuée par tous les élèves des classes observées et par les étudiants participant à la recherche collaborative, soit les trois phrases graphiques que nous avons analysées dans le tableau précédent. Nous avons sélectionné des extraits d'écrits pour nous représentatifs des tendances ou des éléments réitérés de copie en copie. Au niveau quantitatif, concernant le corpus des élèves, nous avons constitué un souscorpus de 60 écrits à partir des 150 écrits initiaux et, concernant le corpus des étudiants, nous avons conservé l'intégralité des 55 écrits. Nous avons mis ces extraits en regard avec les conseils élaborés à la suite de ces réécritures. L'orthographe des réécritures et des conseils rédigés a été respectée.

À quels éléments syntaxiques et sémantiques de l'écrit proposé les élèves et les étudiants ont-ils été sensibles? Quelles sont les modifications apportées ? Nous allons successivement présenter nos analyses des deux corpus collectés, celui des élèves (en 3) et celui des étudiants de Master 1 (en 4), en insistant sur les points saillants de nos résultats. Notre attention se portera plus particulièrement sur trois axes : la nature des bornes (signes de ponctuation ou connecteurs) ajoutées, le traitement, en relation également à un problème 
de démarcation, du syntagme prépositionnel jusqu'au jour où elle rencontra un petit garçon prénommé Matthieu, ainsi que le traitement des syntagmes nominaux démonstratifs cette maison et ce grand bruit ${ }^{15}$.

Notre objectif est de comparer les réécritures des élèves des classes de la recherche collaborative. Les axes déterminés seront aussi déclinés dans les productions des étudiants se destinant à devenir professeurs des écoles.

\section{Les compétences syntaxiques et sémantiques acquises et les difficultés des élèves d'école primaire et de collège observés dans l'acte de réécriture}

Nous pouvons nous livrer à un premier constat : pour la plupart des élèves observés, la tâche de réécriture s'est limitée à ponctuer différemment le texte, et à cela seulement; la consigne était pourtant de réécrire le texte. Nous émettons l'hypothèse que le fait d'avoir travaillé sur les signes de ponctuation avant ce travail de réécriture est susceptible d'avoir favorisé ce parti pris. Toutefois, les réécritures illustrent une lecture critique de l'écrit observé qui se révèle également dans les conseils de réécriture formulés par les élèves lecteurs de la copie.

\subsection{Les bornes ajoutées}

La plupart des élèves sont sensibles à l'absence de bornes entre les deux premières phrases syntaxiques. Ainsi, ces deux premières phrases ont été souvent séparées, après Jenny, par un signe de ponctuation forte, ou encore par une virgule interphrastique comme dans l'exemple suivant :

[Il était une fois, une jeune fille qui s'appelait Jenny, // elle habitait dans cette maison depuis longtemps. //]PG1 (M., $4^{\text {ème }}, 13$ ans)

Des virgules ont été abondamment ajoutées en relecture alors que, dans une situation d'écriture, il s'agit d'un signe très complexe à employer (Paolacci, Dufour \& Bain, 2016 ; Paolacci \& Rossi-Gensane, 2019). L'exemple suivant de C. (CM1, 9 ans) montre un attrait particulier pour ce signe, notamment dans un emploi interphrastique (quatre ajouts aux frontières des phrases syntaxiques) :

[Il était une fois, une jeune fille qui s'apelait Jenny, // elle habitait dans cette maison depuis longtemps. //]PG1 [Jusqu'au jour où elle rencontra un pettit garçon prénommé Matthieu, // ils passaient beaucoup de temps ensemble. //]PG2 [Un jour Jenny et Matthieu allaient jouer dehors en pleine nuit, // ils allèrent dans un bois, // soudain il se retourna en entendant ce grand bruit. //]PG3

Il est intéressant d'apprécier les conseils de réécriture de l'élève C. appuyant son choix du signe de la virgule : Un petit conseil pour tes phrase: Il faut que tu mette de phrase moins longue ou si tu mets des phrase longue met plus de virgule.

De plus, nous avons observé un attachement à la virgule après Il était une fois. Dans la classe de $\mathrm{CE} 2 / \mathrm{CM} 1 / \mathrm{CM} 2$ (élèves de 8 à 11 ans), $78 \%$ des élèves insèrent une virgule après cette expression qui, composée d'un présentatif et d'un syntagme nominal fournissant une indication de temps, n'est pourtant pas un constituant. Nous pouvons émettre l'hypothèse qu'à cet endroit-là, une pause est marquée en lecture à haute voix et que cette pause induit la présence de la virgule. En effet, nous avons remarqué dans notre recherche que la fonction intonative de la ponctuation était l'une des fonctions particulièrement plébiscitées par les élèves (Jaffré, 2014). Par ailleurs, a également pu concourir à l'isolement de cette expression, qui introduit un conte, son rôle de marqueur de genre textuel.

Certains élèves font preuve d'inventivité en proposant un large spectre de signes de ponctuation, tels les points d'exclamation, les points de suspension (comme l'illustre 
l'élève E. de $6^{\text {ème }}$ cité infra en 3.3$)$ ou les deux points. L'élève N. ( ${ }^{\text {ème }}, 13$ ans et demi) emploie à trois reprises les points d'exclamation à la fin des phrases graphiques 4,6 et 7 (cette dernière phrase, imposée, ayant été modifiée) :

[Il était une fois, une jeune fille qui s'appelait Jenny. //]PG1 [Elle habitait dans cette maison depuis longtemps. I/]PG2 [Jusqu'au jour où elle rencontra un petit garçon prénommé Matthieu. //]PG3 [Ils passaient beaucoup de temps ensemble! //]PG4 [Un jour Jenny et Matthieu allaient jouer dehors en pleine nuit, // ils allèrent dans un bois. //]PG5 [Soudain!]PG6 [Il se retourna en entendant ce grand bruit! //]PG7

\subsection{Le traitement de l'apparent complément circonstanciel de temps " jusqu'au jour où elle rencontra un petit garçon prénommé Matthieu »}

Certains élèves isolent le syntagme prépositionnel dans une phrase graphique, ce qui a pour effet de mettre en conformité phrase graphique et phrase syntaxique, même s'il s'agit d'une sorte très particulière de phrase syntaxique. L'élève G. (CM2, 11 ans) propose, d'une certaine façon, une solution intermédiaire en recourant au signe de ponctuation moyenne (rare) qu'est le point-virgule et, dans sa réécriture, illustre des compétences démarcatives abouties. Comme le montre son travail ci-dessous, quatre points et trois virgules ont été ajoutés, dont des virgules intraphrastiques après les organisateurs temporels (ce qui est peu fréquent dans le corpus). Six phrases graphiques pour sept phrases syntaxiques peuvent être dégagées :

[Il était une fois, une jeune fille qui s'appelait Jenny. //]PG1 [Elle habitait dans cette maison depuis longtemps; // jusqu'au jour où elle rencontra un petit garçon prénommé Matthieu. //]PG2 [Ils passaient beaucoup de temps ensemble. //]PG3 [Un jour, Jenny et Matthieu allaient jouer dehors en pleine nuit. //]PG4 [Ils allèrent dans un bois. //]PG5 [Soudain, il se retourna en entendant ce grand bruit. //]PG6

Mis à part le cas de l'apparent complément différé, on note une coextensivité entre phrases graphiques et phrases syntaxiques, souvent signe de phrases graphiques courtes, valorisées dans les rédactions d'école primaire depuis au moins la fin du XIXe siècle (voir Chervel, 2008).

\subsection{Le traitement des syntagmes " cette maison 》 et " ce grand bruit 》}

Certains élèves de $4{ }^{\text {ème }}$ relèvent le problème de cohérence du début du texte (nous l'avons observé dans leurs annotations) mais ne modifient pas le texte lors de la réécriture. Ainsi, l'élève A. ( $4^{\text {ème }}, 13$ ans) entoure elle habitait dans cette maison depuis longtemps et écrit: Ça ne colle pas avec le début de la phrase ou changer cette par une. Il entoure aussi il se retourna et commente : ses plutôt ils se retournèrent car ils sont deux. Ces commentaires prouvent que cet élève est attentif aux effets de cohérence de l'incipit.

Un autre élève de collège, Ma. ( $6^{\text {ème }}, 12$ ans), apporte également dans ses conseils toute son attention à la question de la cohérence textuelle : De quel maison tu parles? Tu aurait du mettre elle habitait dans cette maison depuis longtemps après la rencontre de Matthieu et de Jenny.

Quelques élèves, pour rétablir la cohérence, ont modifié le contenu du texte. L'élève E. $\left(6^{\text {ème }}, 12\right.$ ans) barre, à l'intérieur des phrases imposées, un segment (dans cette maison, mais aussi il se retourna) et y substitue immédiatement un autre qui diffère en un (seul) point (ici, respectivement, l'article indéfini et le pluriel) :

[Il était une fois, une jeune fille qui s'appelait : Jenny // elle habitait dans centen dans une maison // et elle y vivait depuis très longtemps. //]PG1 [Jusqu'au jour où... elle rencontra un petit garçon prénommé: Matthieu // ils passaient beaucoup de temps ensemble. //]PG2 [Un jour Jenny et Matthieu allaient jouer dehors en pleine nuit, // ils 
allèrent dans un bois // soudain... il se retouna ils se retournèrent en entendant ce grand bruit. //]PG3

Cette pratique d'opérations de réécriture, dite de remplacement, comme d'ailleurs celles d'ajout ${ }^{16}$ ou de suppression, reste peu exploitée en première écriture par les élèves dans le corpus des «trois phrases », ainsi que le note Garcia-Debanc (2018). En cela, l'élève E. témoigne d'une lecture minutieuse de la production.

\section{Les compétences syntaxiques et sémantiques acquises et les difficultés des étudiants de Master 1 MEEF observés dans l'acte de réécriture}

Pour le corpus des écrits d'étudiants, nous illustrons les trois mêmes axes que ceux analysés pour les élèves.

\subsection{Les bornes ajoutées}

Tous les étudiants observés résolvent le phénomène de sous-ponctuation entre les deux premières phrases syntaxiques identifiées plus haut dans le cadre de la première phrase graphique : [Il était une fois une jeune fille qui s'appelait Jenny // elle habitait dans cette maison depuis longtemps. //]PG1. Ils insèrent pour la plupart une ponctuation forte sous la forme d'un point.

Concernant les emplois des virgules, nous notons que 36\% des écrits des étudiants comportent l'ajout d'une virgule après Il était une fois, comme dans l'extrait suivant où, de plus, l'étudiant ${ }^{17} 18$ ne respecte pas la consigne car il modifie les phrases imposées pour résoudre la question des référents :

[Il était une fois, une jeune fille qui s'appelait: Jenny. I//PG1 [Elle habitait depuis longtemps dans une maison. //]PG2 [Un jour, elle rencontra un petit garçon prénommé : Matthieu. //]PG3 [Depuis, là, ils passaient beaucoup de temps ensemble. //]PG4 [Jenny et Matthieu, allaient jouer dehors en pleine nuit dans un bois quand soudain, il entendit un grand bruit et se retourna. //]PG5 (étudiant 18)

Cet étudiant abuse des virgules (comme nous l'avons remarqué pour ce niveau d'études dans nos travaux antérieurs (Paolacci \& Rossi-Gensane, 2019)), cf., outre "Il était une fois, », « Depuis, là, » et « Jenny et Matthieu, allaient ». Dans ce dernier cas, une virgule sépare sujet et pivot verbal, ce qui n'est plus conforme à la norme, qui a toutefois " autorisé pendant longtemps l'introduction d'une virgule immédiatement après le sujet à partir du moment où celui-ci était d'une certaine longueur » (Dürrenmatt, 2015 : 60). On comprend mieux cette tendance de l'étudiant quand on lit ses conseils : L'élève doit essayer de lire son texte et doit placer des virgules ou des points au moment où elle doit reprendre sa respiration. Pour cet étudiant, la fonction intonative de la ponctuation prime, et non la fonction syntaxique, comme l'indique d'ailleurs l'absence de balance, c'est-à-dire de ponctuation double (voir Catach, 1994 : 43), à l'endroit de soudain (à l'inverse de là). En effet, soudain est seulement affecté d'une virgule fermante alors qu'il devrait être encadré par deux virgules signalant ici, sur un plan syntaxique, un constituant présenté comme accessoire. L'étudiant 21 accorde aussi la primauté à la fonction intonative dans ses conseils : Pour la ponctuation, quand tu relis, lorsque tu marques une pause, il faut mettre un point ou une virgule.

Certains étudiants, au contraire, ne recourent à aucune virgule dans toute leur réécriture, même après les organisateurs temporels, comme le montre l'extrait suivant :

[Il était une fois une jeune fille qui s'appelait Jenny. I/]PG1 [Elle habitait dans cette maison depuis longtemps quand elle rencontra un petit garçon prénommé mathieu. //]PG2 [Ils passaient beaucoup de temps ensemble. //]PG3 [Une nuit mathieu et Jenny allèrent 
jouer dehors. //]PG4 [Mathieu jouait dans les bois quand soudain il se retourna en entendant ce grand bruit. //]PG5 (étudiant 20)

La réécriture de ce dernier étudiant est en contradiction avec les commentaires qu'il écrit: La ponctuation est légère de nombreuse phrase un peu longue mairiterais des virgule. De plus les seul signe utilisé sont le point et la virgule. Il manque un certain nombre de point.

D'autres signes de ponctuation sont parfois employés. Ainsi, l'étudiant 18, dans l'écrit supra, semble s'essayer aux deux points.

\subsection{Le traitement de l'apparent complément circonstanciel de temps " jusqu'au jour où elle rencontra un petit garçon prénommé Matthieu »}

La plupart des étudiants ont remplacé jusqu'au jour où par un jour (voir par exemple l'écrit de l'étudiant 18 supra en 4.1). Certains étudiants comme l'étudiant 3, qui, en outre, transforme cette maison en une maison, ne se sont pas questionnés sur le rattachement du syntagme prépositionnel : Elle habitait depuis longtemps dans une maison jusqu'au jour où elle rencontra un petit garçon prénommé Matthieu (extrait de l'écrit de l'étudiant 3). Notons, là encore, que le conseil de l'étudiant renvoie à la primauté accordée au rôle prosodique de la ponctuation, que nous avons déjà soulignée : Pour écrire ce texte, il faut que tu relises tes phrases dans ta tête ou à haute voix pour repérer les temps de pause et donc où tu dois placer les virgules ou les points.

\subsection{Le traitement des syntagmes " cette maison " et " ce grand bruit "}

Certains étudiants n'ont pas surmonté les obstacles des emplois des déterminants démonstratifs (n'ayant pas vu la nécessité de penser en amont le référent). Néanmoins, nous relevons des écrits plutôt aboutis comme celui de l'étudiant 19, malgré l'absence de virgules après les organisateurs temporels Un jour, Un soir et Soudain de début de phrase : [Il était une fois une jeune fille qui s'appelait Jenny. //]PG1 [Elle vivait dans une maison près de la forêt. //]PG2 [Elle habitait depuis longtemps dans cette maison. //]PG3 [Un jour elle rencontra un petit garçon prénommé Matthieu. //]PG4 [Un soir Jenny et Matthieu allèrent jouer en pleine nuit. //]PG5 [Ils allèrent dans un bois. I/]PG6 [Soudain Matthieu entendit un craquement assourdissant. //]PG7 [Il se retourna en entendant ce grand bruit. //]PG8 (étudiant 19)

Les conseils de réécriture de cet étudiant illustrent une attention particulière portée aux difficultés de l'élève, même si certaines erreurs terminologiques sont présentes $^{18}$ :

Attention, pour pouvoir utiliser le pronom [sic] "ce » il faut que le nom qu'il désigne ait été déjà introduit dans le récit.

Attention au connecteur [sic] "jusqu'au jour » : " elle habitait dans cette maison jusqu'au jour où » signifie qu'après cela elle n'habite plus dans cette maison. [...]

Attention à ne pas écrire des phrases trop longues.

Chez de nombreux étudiants, nous notons l'invitation, réitérée aussi par de nombreux enseignants (Rondelli, 2013), à écrire des phrases courtes ou plus courtes, dans le prolongement de la préconisation de brièveté mentionnée dans une perspective historique par Chervel (2008) (voir supra en 3).

\section{En guise de conclusion, que retenir de cette étude au niveau didactique?}

Selon l'hypothèse que nous avons formulée, la posture de lecteur d'un écrit qui n'est pas le sien accroît également en posture de scripteur la sensibilité à la syntaxe des phrases et à la 
sémantique du texte. Cette posture de lecteur gagne en outre à être renforcée par une posture d'évaluateur-prescripteur, qui incite les élèves à expliciter le ressenti du lecteur par des commentaires métalinguistiques. Autrement dit, les élèves peuvent être amenés au «faire » par le biais du "dire de faire ». Nous en avons l'assurance à la fois dans les réécritures produites et dans les conseils écrits : les élèves et les étudiants observés sont en grande majorité sensibles à l'absence de bornes comme les signes de ponctuation, ainsi qu'aux problèmes de référence liés à certaines expressions.

À l'issue de notre étude, des «styles» de relecteurs (pour reprendre l'expression de Vénérin-Guénez, 2014) peuvent être définis. Certains élèves tels que E. ( $6^{\text {ème }}, 12$ ans) privilégient dans leur réécriture une ponctuation lecturale (Bessonnat, 1991) en utilisant des points de suspension qui amènent le lecteur à interpréter autrement le texte (voir supra en 3.3). Notre corpus a de plus montré que des élèves développaient en réécriture une ponctuation expressive révélatrice d'un effet sur le lecteur potentiel (voir supra en 3.1). Grâce à ce travail mené au sein de la recherche collaborative, dont nous n'avons présenté qu'une petite partie, les élèves observés ont porté une attention particulière à la segmentation en phrases de l'un de leurs camarades et ont été convaincus de l'importance de la ponctuation (une influence de ce travail à moyen terme a pu être évaluée positivement en fin d'année dans les classes). Les problèmes de référence ont été plus difficilement identifiés, ce qui est sans doute peu surprenant pour des élèves d'école primaire; en revanche, ce point serait à travailler avec les classes des élèves plus âgés du collège.

De leur côté, les étudiants ont été mis en situation d'évaluer l'écrit d'un élève mais en ayant pris conscience des obstacles de l'activité rédactionnelle, notamment grâce à l'activité de réécriture. Les conseils de réécriture qu'ils ont prodigués ont permis d'envisager la copie d'une manière positive. De par la comparaison des données recueillies auprès des étudiants avec celles des élèves, nous avons pu relever des similitudes en ce qui concerne les difficultés, comme les emplois non normés de virgules ou la primauté accordée à la fonction intonative de la ponctuation (au détriment des fonctions syntaxique ou sémantique). Tous ces aspects montrent que les étudiants de Master se destinant à devenir professeurs des écoles doivent continuer à asseoir leurs connaissances linguistiques, les apprentissages langagiers devant être considérés sur le long terme.

Dans la deuxième année de la recherche collaborative, les enseignants des classes observées prolongeront la réflexion en mettant en œuvre des rituels autour des emplois des signes de ponctuation. Ces enseignants pour la plupart formateurs sont persuadés de la nécessité de former les enseignants, débutants ou plus expérimentés, à l'enseignement de l'écriture en insistant sur les liens avec l'enseignement de l'étude de la langue (Dolz \& Gagnon, 2018).

\section{Références bibliographiques}

Bally, C. (1944). Linguistique générale et linguistique française. Berne : Francke.

Béguelin, M.-J. (éd.) (2000). De la phrase aux énoncés: grammaire scolaire et descriptions linguistiques. Bruxelles : De Boeck Duculot.

Bessonnat, D. (1991). Enseigner la... « ponctuation » ?(!). Pratiques, 70, 9-49.

Catach, N. (1994). La ponctuation. Paris : Presses Universitaires de France.

Cautela, A. \& Marin, B. (2013). Emprunts et empreintes textuel(le)s. Le français aujourd'hui, 181, $37-45$.

Charolles, M. (2002). La référence et les expressions référentielles en français. Paris : Ophrys.

Chervel, A. (2008). Histoire de l'enseignement du français du XVIIe au XXe siècle. Paris : Retz. 
Cnesco (2018). Écrire et rédiger: comment guider les élèves dans leurs apprentissages ? https://www.cnesco.fr/ecrire-et-rediger/ (consulté le 22 décembre 2019).

Crinon, J. \& Marin, B. (2014). La production écrite, entre contraintes et expression. Paris : Nathan.

David, J. \& Doquet, C. (2016). Des écrits d'élèves, un corpus de référence pour le français contemporain. Actes du $5^{\text {ème }}$ Congrès Mondial de Linguistique Française.

David, J. \& Vaudrey-Luigi, S. (éds) (2014). Enseigner la ponctuation. Le français aujourd'hui, 187.

Dolz, J. \& Gagnon, R. (2018). Former à enseigner la production écrite. Villeneuve d'Asq: Septentrion.

Dürrenmatt, J. (2015). La ponctuation en français. Paris : Ophrys.

Éduscol (2015). https://cache.media.eduscol.education.fr/file/Ecriture/10/3/18_RA_C3_Francais_Ecri ture_REECRIRE_591103.pdf (consulté le 17 novembre 2019).

Fabre-Cols, C. (2002). Réécrire à l'école et au collège. De l'analyse des brouillons à l'écriture accompagnée. Issy-les-Moulineaux : ESF Éditeur.

Favart, M. \& Chanquoy, L. (2007). Les marques de cohésion comme outils privilégiés de la textualisation : une comparaison entre élèves de CM2 et adultes experts. Langue française, 155, 51-68.

Feuillard, C. (1989). La syntaxe fonctionnelle dans le cadre des théories linguistiques contemporaines. Thèse d'État, Université Paris V.

Garcia-Debanc, C. (2018). Ajout et résolution de problèmes de cohésion textuelle : analyses linguistiques de textes d'élèves et présentation de différents dispositifs de travail pour enseigner l'ajout au cycle 3. Repères, 57, 185-208.

Garcia-Debanc, C. \& Bonnemaison, K. (2014). La gestion de la cohésion textuelle par des élèves de 11-12 ans : réussites et difficultés. Actes du $4^{\text {eme }}$ Congrès Mondial de Linguistique Française, 961-976.

Garcia-Debanc, C. \& Bras, M. (2016). Vers une cartographie des compétences de cohérence et de cohésion textuelle dans une tâche-problème de production écrite réalisée par des élèves de 9-12 ans : indicateurs de maitrise et progressivité. In S. Plane, C. Bazerman, F. Rondelli, C. Donahue, A. N. Applebee, C. Boré, P. Carlino, M. Marquilló Larruy, P. Rogers \& D. Russell (éds). Recherches en écritures: regards pluriels. Recherches textuelles, 13. Metz, Université de Lorraine, 39-62.

Gautier, A. (2010). Syntaxe et ponctuation en conflit. Le point est-il une limite de la rection?. Travaux de linguistique, 60, 91-107.

Jaffré, J.-P. (2014). À quoi sert la ponctuation?. In J. David \& S. Vaudrey-Luigi (éds). Le français aujourd'hui, 187, 129-137.

MEN (2018). Programmes du cycle 3. https://cache.media.eduscol.education.fr/file/programmes 201 $\underline{8 / 20 / 2 / \text { Cycle } 3 \text { programme consolide 1038202.pdf }}$

Niwese, M., Lafont-Terranova, J. \& Jaubert, M. (éds) (2019). Écrire et faire écrire dans l'enseignement postobligatoire. Enjeux, modèles et pratiques innovantes. Villeneuve d'Asq: Presses Universitaires du Septentrion.

Paolacci, V., Bain, D. \& Dufour, M.-P. (2016). L'enseignement de la ponctuation. Le cas de la virgule. In S. Chartrand (éd.). Mieux enseigner la grammaire. Pistes didactiques et activités. Québec : ERPI. 225-248.

Paolacci, V. \& Rossi-Gensane, N. (2012). Quelles images de la phrase dans les écrits d'élèves de fin d'école primaire française? Description linguistique et réponses didactiques aux difficultés des

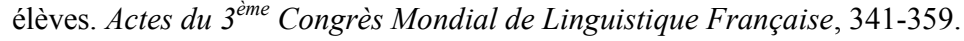


Paolacci, V. \& Rossi-Gensane, N. (2014). Ponctuation et écrits d'élèves : une conception différente de la phrase pour enseigner la ponctuation autrement. In J. David \& S. Vaudrey-Luigi (éds). Le français aujourd'hui, 187, 115-125.

Paolacci, V. \& Rossi-Gensane, N. (2016). La question de la progressivité des apprentissages en production écrite à l'école élémentaire française : le cas de la segmentation en phrases. Actes $d u$ $5^{\text {ème }}$ Congrès Mondial de Linguistique Française.

Paolacci, V. \& Rossi-Gensane, N. (2019). Les emplois de la virgule dans des écrits d'étudiants se destinant à devenir enseignants. In M. Niwese, J. Lafont-Terranova \& M. Jaubert (éds). Écrire et faire écrire dans l'enseignement postobligatoire. Enjeux, modèles et pratiques innovantes. Villeneuve d'Asq : Presses Universitaires du Septentrion. 213-229.

Riegel, M., Pellat, J.-C. \& Rioul, R. (2009). Grammaire méthodique du français. Paris : Presses Universitaires de France ( $1^{\text {re }}$ édition : 1994).

Rondelli, F. (2013). La phrase, segment textuel «de base »? Choix d'écriture d'élèves de cycle 3 et jugements des enseignants. Le français aujourd'hui, 181, 71-81.

Rossi-Gensane, N. \& Paolacci, V. (2016). La segmentation des écrits d'élèves à l'école et au collège : quel rôle pour la phrase ?. In C. Boré (éd.). LIDIL, 54, 35-53.

Rossi-Gensane, N., Roubaud, M.-N. \& Paolacci, V. (2019). La phrase à l'épreuve de la complexité à l'école élémentaire. La phrase, carrefour linguistique et didactique, Actes du $10^{\text {ème Colloque }}$ International de linguistique française et roumaine, Arras, 3-5 juin 2015. Arras : Artois Presses Université. 325-347.

Vénérin-Guénez, C. (2014). À la recherche de styles de scription. Usages de la ponctuation : erreurs, nomes, créativité. In J. David \& S. Vaudrey-Luigi (éds). Le français aujourd'hui, 187, 67-81.

Vetters, C. (1993). Passé simple et imparfait : un couple mal assorti. Langue française, 100, 14-30.

\section{Annexe}

\section{Copie d'un élève d'une classe de $6^{\text {ème }}$}

(Seule l'orthographe a été rectifiée.)

Il était une fois une jeune fille qui s'appelait Jenny elle habitait dans cette maison depuis longtemps. Jusqu'au jour où elle rencontra un petit garçon prénommé Matthieu ils passaient beaucoup de temps ensemble. Un jour Jenny et Matthieu allaient jouer dehors en pleine nuit, ils allèrent dans un bois soudain il se retourna en entendant ce grand bruit. Ils coururent mais ils s'enfoncèrent de plus en plus dans le bois, au bout d'une heure de course ils étaient perdus, et frigorifiés. Mais le bruit était encore là les enfants eurent de plus en plus peur mais ne pouvaient plus partir ils étaient résignés à attendre, le bruit se rapprocha encore jusqu'à être dans la lumière de la nuit. Ce bruit était en fait un petit ours blessé qui cherchait ses parents les deux enfants le prirent au bout de deux heures de marche ils arrivèrent enfin chez eux, puis ils réveillèrent toute la maison. La maman de Jenny dit à Matthieu de rentrer chez lui et qu'ils reparleraient de tout ça demain pour le moment il fallait soigner le petit ours. Le lendemain le petit ours put rentrer chez lui avec l'aide des parents de Jenny. Un peu plus tard la mère de Jenny invita les parents de Matthieu pour leur parler de ce qui s'était passé la veille, au bout de X temps ils décidèrent de décréter que depuis cette aventure les enfants ne sortent plus la nuit mais ça n'a pas empêché Matthieu et Jenny de se revoir et de jouer.

\section{FIN}

\footnotetext{
${ }^{1}$ Nous remercions les relecteurs de leurs commentaires.

2 Selon Riegel, Pellat et Rioul (2009: 1020), «la cohésion d'un texte [...] dépend de facteurs sémantiques et syntaxiques qui assurent son unification ", tels, notamment, les procédés de reprise anaphorique et les connecteurs, auxquels les psycholinguistes ajoutent,
} 
du fait de leur lien étroit avec ces derniers, les signes de ponctuation (voir, par exemple, Favart \& Chanquoy, 2007). Toujours pour Riegel, Pellat et Rioul (2009 : 1019), «la cohérence caractérise la bonne formation interprétative et communicative du discours », même si, "dans la pratique, il est difficile d'opérer une stricte répartition entre des règles de cohérence à portée externe et des règles de cohésion à portée interne ». Cela nous semble particulièrement le cas pour les procédés de reprise anaphorique, d'ordre plus global que les connecteurs et les signes de ponctuation, d'ordre plus local, et à portée à la fois interne et externe, puisqu'ils impliquent le phénomène de référence.

3 https://cache.media.eduscol.education.fr/file/Ecriture/10/3/18 RA_C3 Francais_Ecriture REECRIRE 591103.pdf

${ }^{\overline{4}}$ Nous nous permettons de substituer le terme de pivot à celui de prédicat, polysémique.

${ }^{5}$ Lorsque seule la notion de phrase graphique est utilisée, les virgules sont, conformément à la tradition, nécessairement intraphrastiques.

${ }^{6}$ Voir aussi la journée d'étude du 5 juin 2019 de l'INSPÉ de Paris (Re)lier, connecter, ponctuer : des stratégies pour apprendre à mieux lire et mieux écrire.

7 Nous remercions les membres de la recherche collaborative: Alain Calvet, Maxime Combes, Marie-Pierre Girard, Séverine Isidore-Maviel, Nedjma Lauret-Brehm et Emmanuelle Martin.

${ }^{8}$ Par « écrit de travail », nous entendons un écrit grâce auquel l'élève fixe sa pensée. C'est un écrit transitoire qui vise la mise en mots des acquis de l'élève et qui peut constituer la base d'un oral collectif.

${ }^{9}$ Les formulations « un élève » ou « cet élève » sont à comprendre de manière neutre.

${ }^{10}$ Nous avons présenté la recherche collaborative au colloque AIRDF (Lyon, 26-28 août 2019), dans notre intervention intitulée "Évaluer la segmentation d'écrits d'élèves de l'école et du collège français (élèves de 8 à 14 ans) - Pour quelles aides à la réécriture ?».

${ }^{11}$ La consigne des trois phrases a permis la constitution du corpus Résolco au sein du laboratoire CLLE-ERSS (Garcia-Debanc \& Bras, 2016), qui participe à l'ANR (Agence Nationale de la Recherche) E-CALM dirigée par C. Doquet (David \& Doquet, 2016).

${ }^{12}$ Rappelons que les étudiants français doivent passer un concours pour devenir enseignants tout en validant un Master.

${ }^{13}$ Nous soulignerons les phrases imposées dans les réécritures d'élèves que nous citerons.

14 « Dans l'anaphore conceptuelle [...], l'expression anaphorique ne reprend pas un groupe nominal ou un segment antérieur particulier. Elle condense et résume le contenu d'une phrase, d'un paragraphe ou de tout un fragment de texte antérieur [...]. » (Riegel, Pellat \& Rioul, 2009 : 1038)

${ }^{15}$ Pour une analyse des phénomènes de cohésion et de cohérence d'écrits d'élèves portant sur la consigne des «trois phrases", se reporter également à Garcia-Debanc et Bonnemaison (2014) et à Garcia-Debanc et Bras (2016).

${ }^{16}$ Même s'il ne s'agit pas des éléments plus spécifiquement étudiés, mentionnons brièvement deux ajouts (doublement soulignés) de cet élève à l'endroit de la première phrase imposée : et elle y vivait depuis très longtemps.

${ }^{17}$ Nous écrivons «étudiant » de manière neutre mais il convient de savoir qu'à l'INSPÉ de Toulouse Occitanie-Pyrénées, plus de $80 \%$ des étudiants en Master pour devenir professeurs des écoles sont des femmes.

${ }^{18}$ Dans les conseils de réécriture qui suivent, ce n'est pas un pronom mais un déterminant (l'un des relecteurs remarque une tendance chez de nombreux étudiants à confondre pronom et déterminant, notamment pour les démonstratifs et les possessifs, sans doute en raison de leur côté possiblement anaphorique); le début du syntagme prépositionnel jusqu'au jour n'est pas non plus un connecteur qui relierait deux propositions. 